\title{
Milliliter per Kilogram per Meter Squared
}

National Cancer Institute

\section{Source}

National Cancer Institute. Milliliter per Kilogram per Meter Squared. NCI Thesaurus. Code C120816.

A unit of concentration equal to milliliter divided by kilogram per meter squared. 